\title{
Occurrence and Resistance Pattern of Gram-Negative Bacteremia and Sepsis in A Tertiary Care Hospital - A Four-Year Study
}

\author{
Alamu Juliana* (D), K.V. Leela (D), Anusha Gopinathan (D) and R. Sujith \\ Department of Microbiology, SRM Medical College Hospital \& Research Centre, \\ SRM Institute of Science \& Technology, SRM Nagar, Kattankulathur, Tamil Nadu, India.
}

\begin{abstract}
The rise in antibiotic resistance has been a major source of public health concern. As a result, mortality and morbidity rates have risen significantly. This study was done to identify gram-negative organisms causing bacteremia/sepsis, study their prevalence rates, and antimicrobial resistance patterns, as evidence-based knowledge of gram-negative organisms causing sepsis and their resistance profiles is essential for effective hospital control and better management of infections caused by resistant bacteria. A retrospective study, conducted from January 2016 to December 2019, blood samples were collected using aseptic guidelines and cultured using automated blood culture methods. Biochemical tests were done according to microbiology standard procedures, while antimicrobial testing was done according to CLSI guidelines. A total of 13,808 blood samples were received within the study period of four years. Of the total, 2079 showed significant growth, with 765 being GNB isolates. The most common isolates were Escherichia coli (35.42\%), Klebsiella pneumonia (19.74\%), Acinetobacter species (9.67\%), and other non-fermenting gram-negative bacilli (11.76\%). Escherichia coli showed yearly resistance to aminoglycosides, cephalosporins, penicillin, fluoroquinolones, and B-lactam combination agents. Routine surveillance and awareness of the prevalence, etiological agents, and antibiotic resistance of gram-negative bacteria causing bacteremia/sepsis is critical for individual therapy, hospital control, and the effectiveness of preventive interventions.
\end{abstract}

Keywords: Antimicrobial resistance, Bacteremia, Bloodstream infections, Gram Negative bacteria, Sepsis

*Correspondence: alamukemii@gmail.com

(Received: December 20, 2021; accepted: February 7, 2022)

Abbreviations: GNB-Gram negative bacteria, E.coli - Escherichia coli, KPN - Klebsiella pneumonae, AC - Acinetobacter, spp - Species.

Citation: Juliana A, Leela KV, Gopinathan A, Sujith R. Occurrence and Resistance Pattern of Gram-Negative Bacteremia and Sepsis in A Tertiary Care Hospital-A Four-Year Study. J Pure Appl Microbiol. 2022;16(1):655-662. doi: 10.22207/JPAM.16.1.67

(C) The Author(s) 2022. Open Access. This article is distributed under the terms of the Creative Commons Attribution 4.0 International License which permits unrestricted use, sharing, distribution, and reproduction in any medium, provided you give appropriate credit to the original author(s) and the source, provide a link to the Creative Commons license, and indicate if changes were made. 


\section{INTRODUCTION}

Bloodstream infection (BSI) is the presence of viable microorganisms in the blood, which is clinically demonstrated by the positivity of blood culture. ${ }^{1}$ In healthy individuals, the blood is sterile. ${ }^{2}$ Nevertheless, localized infections in a specific body site, such as pneumonia, urinary tract infection, or meningitis, can cause microbial invasions into the bloodstream. Microbes in the bloodstream can proliferate and survive via a variety of mechanisms. Examples are biofilm formation and capsule production. ${ }^{3}$ During the growth and multiplication of microbes in the bloodstream, several toxins are released that cause inflammation and trigger the response of the host. An uncontrolled inflammatory response by the host may lead to sepsis and septic shock. Sepsis is a life-threatening organ dysfunction that is caused by a dysregulated host response to infection. ${ }^{4,25}$ Microorganisms in the blood could either be bacteria, fungi, or viruses. The presence of bacteria in the bloodstream is called bacteremia. ${ }^{5}$ Bacteria that cause bacteremia can be differentiated via gram stain. Bacteria that retain the primary stain are called gram-positive bacteria, while those that retain the secondary stain are called gram-negative bacteria. Time is very crucial in sepsis treatment. However, Due to the lack of a proper rapid identification method, broad-spectrum antibiotics are administered to patients clinically suspected of having sepsis, and this has resulted in the inadvertent administration of broad spectrum antibiotics and an increase in antimicrobial resistance by organisms to almost all available antibiotics. The global prevalence of BSIs varies based on geographical location. ${ }^{6,7}$ Although it is important to note that over the past decade, there has been a notable and significant increase in the world-wide prevalence of antimicrobial resistance gram negative bacterial infections. In developed countries like the United States, approximately 250,000 BSI episodes are recorded yearly, while in developing countries like India, the exact prevalence rate is yet to be established. ${ }^{26,8}$ As per the published reports by the World Health Organization, antimicrobial resistance in gram-negative bacteria is significantly on the increase. This increase is associated with treatment failure, longer hospitalization, and mortality, and thus poses a serious threat to the health care profession. ${ }^{9}$ The importance of regular regional antimicrobial resistance surveillance cannot be overstated in order to effectively control the spread of infections and antimicrobial resistance. Local knowledge of the main pathogens causing bacteremia/sepsis, as well as antimicrobial resistance profiles, will serve as a guide for antimicrobial treatment, as well as provide a better management strategy for antimicrobial resistant strains and empirical therapy for specific pathogens, ultimately improving outcomes. The present study is a single-center study that aims at identifying the various Gram-negative organisms that cause bacteremia and sepsis, their prevalence rate, and antimicrobial resistance pattern.

\section{METHODOLOGY}

\section{Study design and Data collection}

This study is a retrospective study of patients identified with gram-negative bacteremia/ sepsis between January 1, 2016 and December 31, 2019. The study was presented and approved by the ethical committee of our institution (SRM Medical College and Research Center). Patient consent was not required for the study. All blood positive cultures identified with gram negative bacteria during the study period were included, while blood positive cultures identified as either contaminants or gram positive bacteria were excluded. Details like the laboratory number, age, gender, sample collection, date of isolation, organism identified, and organism sensitivity/ resistance to antibiotics were collected by manual observation of laboratory records.

Blood samples were aseptically collected, inoculated, and sent in BacT/ALERT ${ }^{\circledR} 3 \mathrm{D}$ microbial detection culture media bottles upon the clinical suspicion of BSI. Culture media bottles were incubated in the Biomerieux BacT/ALERT ${ }^{\circledR} 3 \mathrm{D}$ microbial detection system until there was a positive signal. Bottles with positive signals were sub-cultured on blood agar, MacConkey agar, and chocolate agar. These plates were incubated for 18-24 hours at $37^{\circ} \mathrm{C}$. Bacteria identification was done using gram staining and standard biochemical tests. Antimicrobial susceptibility testing was done using the Kirby Bauer disc diffusion technique as per Clinical and Laboratory Standards Institute (CLSI) guidelines. ${ }^{24}$ Data entry and analysis were done using IBM SPSS version. ${ }^{25}$ 


\section{RESULTS}

After the exclusion of contaminants, the total number of blood culture positives identified between January 2016 and December 2019 was 2079 (as shown in Table 1).

Our study found a maximum increase in the percentage of blood positives between 2016 and 2017, with the exception of 2018, which had

Table 1. Cases of bacteremia distributed by year

\begin{tabular}{lccccc}
\hline Year & 2016 & 2017 & 2018 & 2019 & Total \\
\hline Positive & 406 & 412 & 368 & 893 & 2079 \\
Negative & 2764 & 2678 & 3079 & 3209 & 11730 \\
Total & 3167 & 3090 & 3447 & 4104 & 13808 \\
\hline
\end{tabular}

the lowest percentage of blood positives, and 2019, which had the highest percentage of blood positives. (Fig. 1).

Fig. 3 and 4 show the gender and age distributions of patients with gram-negative bacteremia/sepsis, respectively. Bacteremia/sepsis was more common in men than in women, with the majority of positive patients aged 50-59 in 2016, and 60-69 in 2017-2019. (Fig. 4).

\section{Isolates Gram Negative Bacteria (GNB)}

All Gram negative bacteria pathogens identified over the study period are shown in Table 2. Between 2016 and 2019, Escherichia coli (35.42\%) was the most prevalent organism, followed by Klebsiella pneumoniae (19.74\%), Acinetobacter species (9.67\%), and other non-

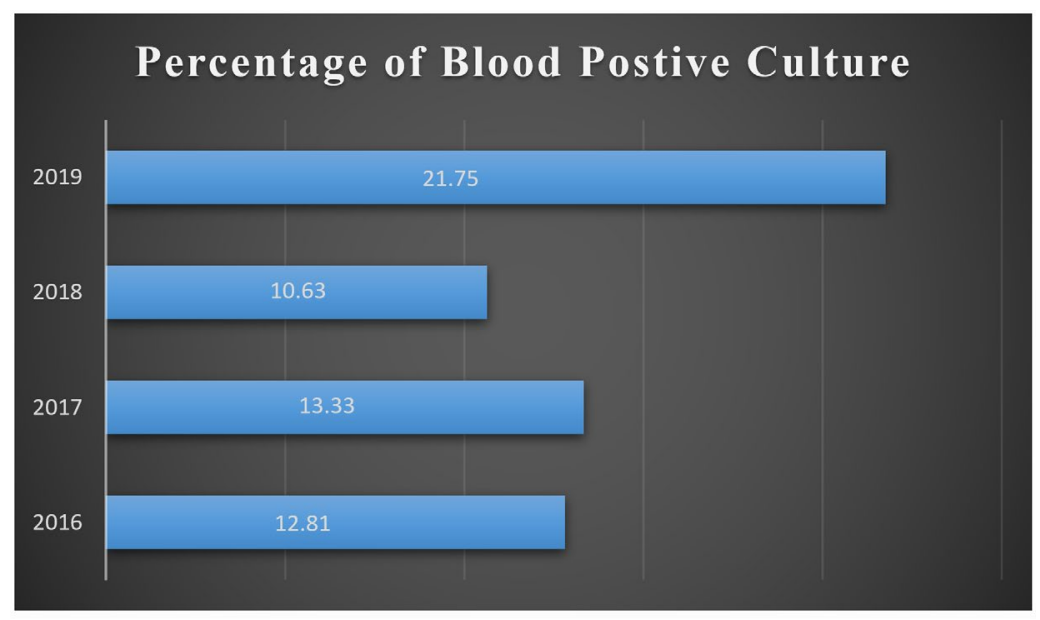

Fig. 1. Shows the year-wise incidence of bacteremia expressed in percentage.

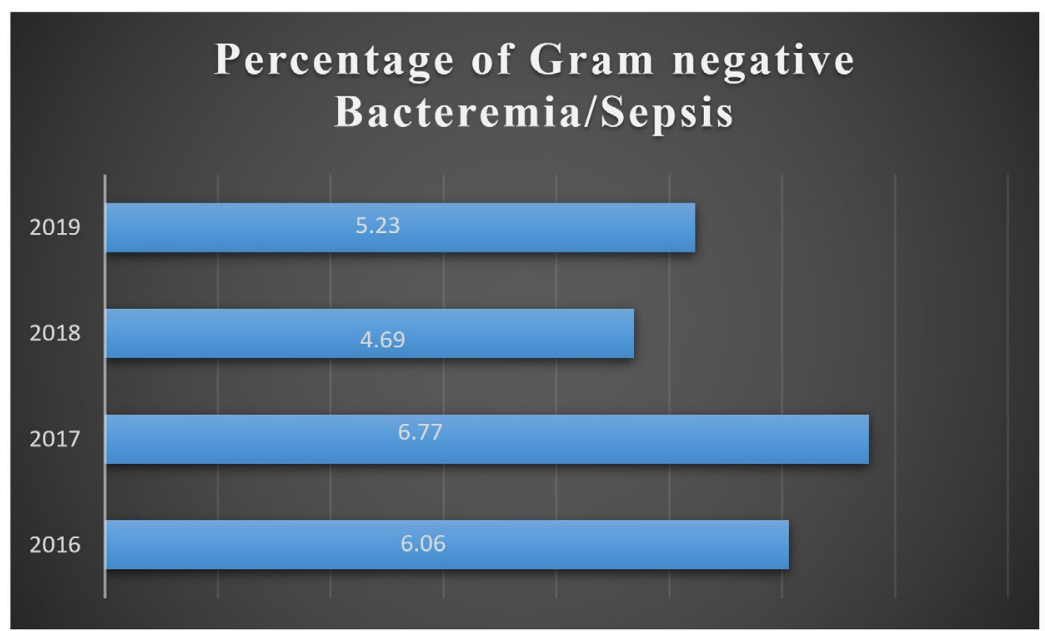

Fig. 2. Shows the year-wise incidence of gram negative bacteremia/sepsis expressed in percentage. 
fermenting gram-negative bacilli (11.76\%). (Table 2).

\section{Antimicrobial Resistance pattern}

The antibiotic resistance patterns of the three (3) most predominant pathogens in this study were examined; they are Escherichia coli, Klebsiella pneumoniae, and Acinectobacter species. Tables 3, 4, and 5 show the resistance patterns of each isolate to the antibiotics examined in our study.

\section{DISCUSSION}

Gram-negative bacteremia/sepsis is a serious, life-threatening challenge that needs prompt action. Due to the lack of proper rapid identification methods, broad-spectrum antibiotics are administered to patients clinically suspected of having sepsis to stabilise them even before the availability of laboratory reports. This, however, has led to the excess use of antibiotics and wide spread resistance, thus causing treatment failure among

Table 2. Etiology of GNB bacteremia /sepsis

\begin{tabular}{lcccc}
\hline Pathogen & $\begin{array}{c}2016 \\
\mathrm{n}=191, \\
\mathrm{n}(\%)\end{array}$ & $\begin{array}{c}2017 \\
\mathrm{n}=205, \\
\mathrm{n}(\%)\end{array}$ & $\begin{array}{c}2018 \\
\mathrm{n}=160, \\
\mathrm{n}(\%)\end{array}$ & $\begin{array}{c}2019 \\
\mathrm{n}=209, \\
\mathrm{n}(\%)\end{array}$ \\
\hline Escherichia coli & $60(31.41)$ & $48(23.41)$ & $73(45.62)$ & $90(43.06)$ \\
Pseudomonas aeruginosa & $24(12.56)$ & $17(8.29)$ & $8(5.00)$ & $5(2.39)$ \\
Acinetobacter spp & $21(10.99)$ & $19(9.26)$ & $11(6.87)$ & $23(11.00)$ \\
Salmonella spp & $19(9.94)$ & $19(9.26)$ & $8(5.00)$ & $20(9.56)$ \\
Klebsiella pneumoniae & $27(14.13)$ & $44(21.46)$ & $34(21.25)$ & $46(22.00)$ \\
Citrobacter spp & $12(6.28)$ & $11(5.36)$ & $5(3.12)$ & $5(2.39)$ \\
Proteus mirabilis & $1(0.50)$ & $6(2.92)$ & $2(1.25)$ & $4(1.91)$ \\
Enterobacter spp & $0(0.00)$ & $5(2.43)$ & $2(1.25)$ & $2(0.95)$ \\
Aeromonas spp & $0(0.00)$ & $3(1.46)$ & $0(0,00)$ & $1(0.47)$ \\
Other non-fermenter & $27(14.13)$ & $33(16.09)$ & $17(10.62)$ & $13(6.22)$ \\
\hline
\end{tabular}

$\mathrm{n}=$ Number of isolates.

Table 3. Shows the resistance pattern of Escherichia coli to antibiotics used during the study period

\begin{tabular}{lcccc}
\hline & \multicolumn{3}{c}{ Escherichia coli } \\
\hline Antibiotics & \multicolumn{4}{c}{ No. of resistant strains (\%) } \\
\cline { 2 - 5 } & 2016 & 2017 & 2018 & $7(9.00)$ \\
\hline Amikacin & $3(5.45)$ & $1(2.22)$ & $6(11.54)$ & $36(52.00)$ \\
Amoxicillin c & $50(93.75)$ & $30(90.91)$ & $60(93.75)$ & \\
lavulanic acid & & & & $62(83.00)$ \\
Ampicillin & $50(96.15)$ & $35(89.74)$ & $56(94.92)$ & $57(66.00)$ \\
Cefotaxime & $50(84.75)$ & $36(80.00)$ & $55(79.71)$ & $48(72.00)$ \\
Ciprofloxacin & $53(86.89)$ & $36(80.00)$ & $61(89.71)$ & $7(8.00)$ \\
Gentamicin & $20(34.84)$ & $17(36.96)$ & $36(49.34)$ & $29(34.00)$ \\
Imipenem & $3(5.45)$ & $1(2.17)$ & $5(6.85)$ & $34(47.00)$ \\
Trimethoprim/ & $46(74.19)$ & $27(65.85)$ & $49(68.06)$ \\
sulfamethoxazole & & & & \\
Cefepime & $45(81.82)$ & $33(76.74)$ & $48(71.64)$ & $52(62.00)$ \\
Ertapenem & $6(10.34)$ & $1(2.27)$ & $10(16.67)$ & $4(6.00)$ \\
Meropenem & $1(1.67)$ & $1(2.27)$ & $5(6.94)$ & $6(7.00)$ \\
Piperacillin/ & $5(8.62)$ & $1(2.33)$ & $9(12.33)$ & $10(11.00)$ \\
Tazobactam & & & &
\end{tabular}


sepsis patients over time. To properly control and manage the increase in antimicrobial resistance, geographical surveillance on the prevalence rate, etiological agents, and antimicrobial resistance pattern of organisms causing bacteremia/sepsis is fundamental for effective treatment.

This study shows the prevalence of gram-negative bacteremia/sepsis and evaluates its resistance profile to the most commonly used antibiotics between January 2016 and December 2019. In our study, the percentage of blood culture positive was $15.05 \%$. This can be compared to the findings of Roy et al. (16.04\%). Meshram et al. $(17.18 \%),{ }^{12}$ Banik et al. (14.24\%). ${ }^{2}$ A higher positive culture rate was recorded by other authors like
Mehdinejah et al. (25.3\%). ${ }^{14}$ and Sharma et al $(33.94 \%) .{ }^{13}$ Variation in the percentage of blood culture positive could be due to geographical and etiological differences, techniques of isolation in different sets up, previous consumption of antibiotics before blood culture, population difference, or number or volume of blood. ${ }^{15,27}$ The clinical significance of studying the percentage of blood positives is that they serve as a strategic tool for monitoring either an increase or decrease in bloodstream infections in a healthcare setting.

Our studies indicate that the prevalence of bacteremia/sepsis was significantly higher in patients aged 50 to 69 , with males having a higher prevalence than females, implying that this age

\section{Incidence Rate For Sex}

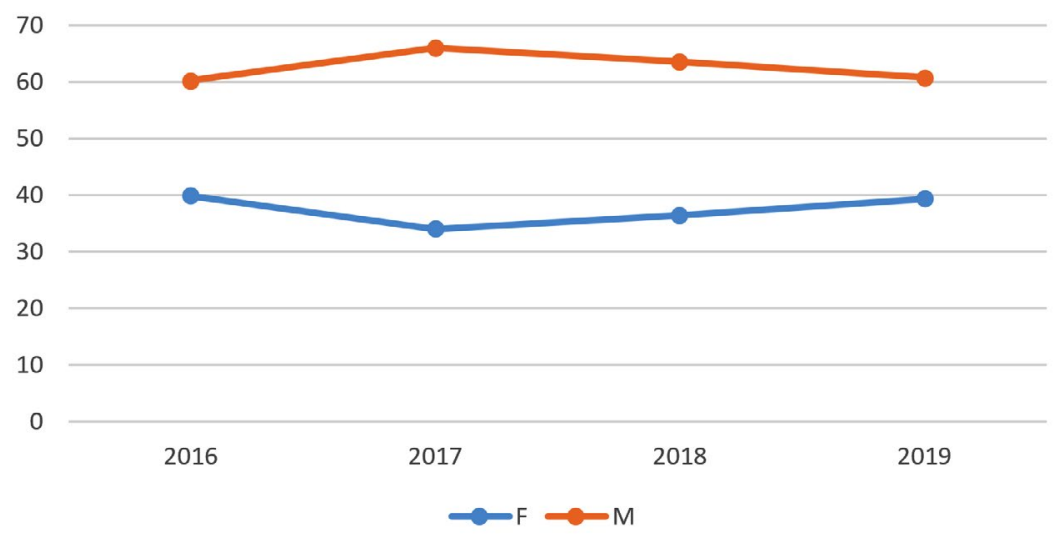

Fig. 3. Shows the incidence of gram negative bacteremia by study year associated with the sex of patients involved..

\section{Incidence by Age Group}

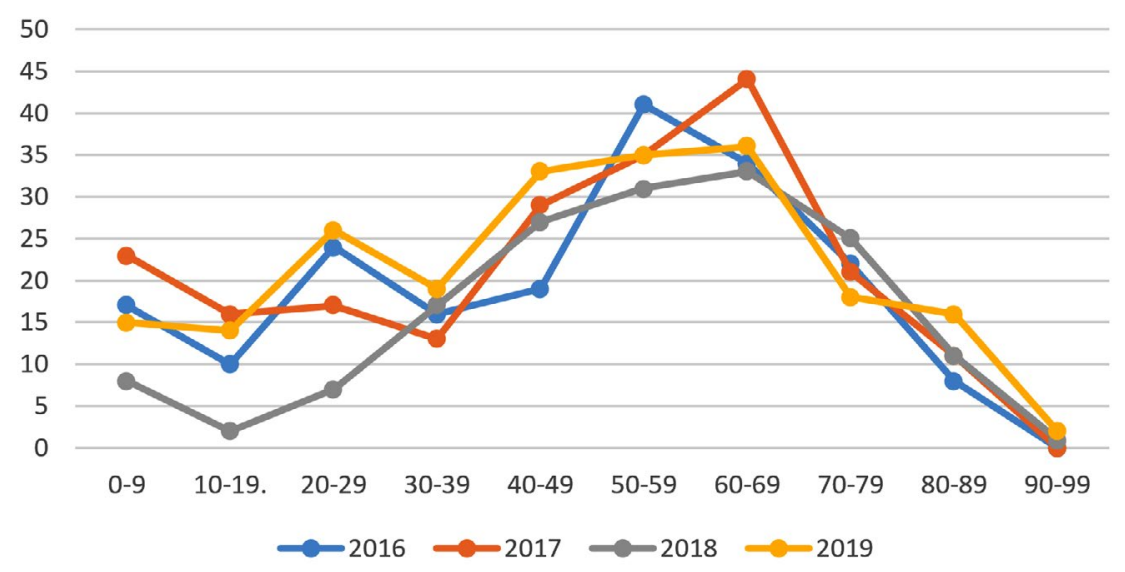

Fig. 4. Shows the incidence of gram negative bacteremia by study year associated with the age of patients involved. 
Juliana et al. | J Pure Appl Microbiol | 16(1):655-662 | March 2022 | https://doi.org/10.22207/JPAM.16.1.67

Table 4. Shows the resistance pattern of Klebsiella pneumoniae to antibiotics used during the study period

\begin{tabular}{lcccc}
\hline & \multicolumn{5}{c}{ Klebsiella pneumoniae } \\
\hline Antibiotics & \multicolumn{4}{c}{ No. of resistant strains (\%) } \\
\cline { 2 - 5 } & 2016 & 2017 & 2018 & $13(31.71)$ \\
\hline Amikacin & $17(28.00)$ & $10(25.64)$ & $7(24.14)$ & $25(59.52)$ \\
Cefotaxime & $21(84.00)$ & $29(72.50)$ & $20(60.61)$ & $21(63.64)$ \\
Ciprofloxacin & $14(66.67)$ & $22(59.46)$ & $11(40.74)$ & $15(36.59)$ \\
Gentamicin & $9(34.62)$ & $19(43.18)$ & $12(35.29)$ & $12(28.57)$ \\
Imipenem & $6(26.09)$ & $6(15.38)$ & $7(21.88)$ & $15(42.86)$ \\
Trimethoprim/ & $17(65.38)$ & $20(50.00)$ & $12(36.36)$ & \\
sulfamethoxazole & & & & $24(58.54)$ \\
Cefepime & $19(76.00)$ & $31(72.09)$ & $16(51.61)$ & $15(39.47)$ \\
Ertapenem & $14(37.04)$ & $14(35.00)$ & $10(38.46)$ & $14(31.82)$ \\
Meropenem & $7(28.00)$ & $10(25.00)$ & $9(28.13)$ & $15(34.88)$ \\
Piperacillin/ & $10(37.04)$ & $12(30.00)$ & $7(20.59)$ \\
Tazobactam & & & & \\
\hline
\end{tabular}

Table 5. Shows the resistance pattern of Acinetobacter spp. to antibiotics used during the study period

\begin{tabular}{lcccc}
\hline \multicolumn{5}{c}{ Acinetobacter spp. } \\
\hline Antibiotics & \multicolumn{5}{c}{ No. of resistant strains (\%) } \\
\cline { 2 - 5 } & 2016 & 2017 & 2018 & $9(39.13)$ \\
\hline Amikacin & $8(38.10)$ & $8(42.11)$ & $6(66.67)$ & $8(40.00)$ \\
Ciprofloxacin & $10(52.63)$ & $8(47.06)$ & $5(50.00)$ & $10(50.00)$ \\
Gentamicin & $8(38.10)$ & $8(42.11)$ & $4(44.44)$ & $8(36.36)$ \\
Imipenem & $10(47.62)$ & $8(42.11)$ & $6(60.00)$ & $7(43.75)$ \\
Trimethoprim/ & $0(0.00)$ & $0(0.00)$ & $0(0.00)$ & $8(40.00)$ \\
sulfamethoxazole & & & & $9(39.13)$ \\
Cefepime & $12(66.67)$ & $9(75.00)$ & $5(62.50)$ & $8(34.78)$ \\
Meropenem & $10(47.62)$ & $8(44.44)$ & $6(60.00)$ & \\
Piperacillin/ & $9(42.86)$ & $7(36.84)$ & $5(50.00)$ & \\
Tazobactam & & & & \\
\hline
\end{tabular}

group requires special attention and preventative strategies to reduce the incidence of bacteremia/ sepsis. A cursory review of the literature for reasons for the higher occurrence of sepsis in males than females reveals that the causes are likely multifactorial, although behavioural, physiological, and immunological factors are all likely to play a part. Our findings are in line with those of Vasudeva et al. ${ }^{16}$ and Santella et al., ${ }^{17}$ which also had a higher prevalence of BSI in males than females in their studies. This is in contrast to that of Zenebe et al. ${ }^{18}$ who found a higher prevalence of BSI in females than in males.

The most common gram-negative bacteria were Escherichia coli (35.42\%), Klebsiella pneumonia (19.74\%), and Acinetobacter species (9.67\%). 2019 had the highest prevalence of Escherichia coli, followed by 2018. These findings are consistent with previous research from NIMS Jaipur ${ }^{16}$ and a few studies from other countries, ${ }^{17,19,20}$ but they differ from Meshram et al. ${ }^{12}$ and Sharma et al. ${ }^{13}$ who discovered a higher prevalence of Klebsiella pneumoniae.

We observed that Escherichia coli was resistant to aminoglycosides, cephalosporins, penicillin, fluoroquinolones, and B-lactam combination agents. (See Table 3). Our findings are congruent with those of Santella et al. ${ }^{17}$ Nagarjuna et al. ${ }^{22}$ and Cole et al. ${ }^{21}$ who discovered maximum resistance to cephalosporins, fluoroquinolones, 
and the penicillin group. A significant rise in carbapenem resistance (i.e., Imipenem and meropenem) was also observed in this study, with $5.45 \%$ and $1.67 \%$ initial resistance in 2016 , but rising to $8.00 \%$ and 7.00 , respectively, in 2019. Klebsiella pneumoniae was resistant to cephalosporins, fluoroquinolones, and trimethoprim/sulfamethoxazole. Antibiotic resistance can be slowed via timely detection, proper infection control measures, periodic surveillance of resistance patterns, and judicious use of antibiotics.

\section{CONCLUSION}

Routine surveillance and awareness of the prevalence, etiological agents, and antibiotic resistance of gram-negative bacteria causing bacteremia/sepsis is critical for individual therapy and hospital control. According to our findings, Escherichia coli was the most common cause of gram-negative bacteremia/sepsis. In general, each year had a high prevalence of Escherichia coli, Klebsiella pneumoniae, and other non-fermenters. Maximum antimicrobial resistance was observed for cephalosporins, penicillin, and the carbapenem group of antibiotics. Antibiotic use should only be encouraged once antimicrobial susceptibility testing has been completed.

\section{ACKNOWLEDGMENTS}

None.

\section{CONFLICT OF INTEREST}

The authors declare that there is no conflict of interest.

\section{AUTHORS' CONTRIBUTION}

All authors listed have made a substantial, direct and intellectual contribution to the work, and approved it for publication.

\section{FUNDING}

None.

\section{DATA AVAILABILITY}

The datasets generated during and analysed during the current study are available from the corresponding author on reasonable request.

\section{ETHICS STATEMENT}

The study was approved by Institutional Ethics committee SRM medical college and Research center, Chennai, India with reference number 2196/IEC/2020.

\section{REFERENCES}

1. Viscoli C. Bloodstream infections: The peak of the Iceberg. Virulence. 2016;(3):248-251. doi: 10.1080/21505594.2016.1152440

2. Banik A, Bhat SH, Kumar A, Palit A, Snehaa K. Bloodstream infections and trends of antimicrobial sensitivity patterns at Port Blair. J Lab Physicians 2018;10(3):332-337. doi: 10.4103/JLP.JLP_50_18

3. Minasyan H. Sepsis: mechanisms of bacterial injury to the patient. Scand J Trauma Resusc Emerg Med. 2019;27(1):19. doi: 10.1186/s13049-019-0596-4

4. Ramachandran G. Gram-positive and gram-negative bacterial toxins in sepsis; a brief review. Virulence. 2014;5(1):213-218. doi: 10.4161/viru.27024

5. Curry WJ, Lewis PR. Bacteraemia and Sepis in: Taylor RB, David AK, Fields SA, Philips DM, Scherger JE. 2003; (eds) Family Medicine, Springer. New York, NY. doi: 10.1007/978-0-387-21744-4_43

6. Zhu Q, Yue $Y$, Zhu L, et al. Epidemiology and microbiology of Gram-positive bloodstream infections in a tertiary care hospital in Beijing, China: a 6 year retrospective study. Antimicrob Resist Infect Control. 2018;7(1):107. doi: 10.1186/s13756-018-0398-x

7. Bajaj A, Mishra B, Loomba PS, et al. Prevalence of Gram negative septicemia in a tertiary care center. J Med SCi Health. 2019;7(3):36-41. doi: 10.46347/JMSH.2019. v05i01.007

8. Khurana S, Bhardwaj N, Kumari M, Mathotra R, Mathur P. Prevalence, etiology and antimicrobial resistance profiles of bacterial bloodstream infections in a tertiary care hospital in Northern India: A 4-year study. J Lab Physicians. 2018;10(4):426-431. doi: 10.4103/JLP. JLP_78_18

9. Breijyeh Z, Jubeh B, Karaman R. Resistance of Gramnegative Bacteria to Current Antibacterial Agents and Approaches to Resolve it. Molecules. 2020;25(6):1340. doi: $10.3390 /$ molecules 25061340

10. Fahim NAE. Prevalance and antimicrobial susceptibility Profile of multidrug-resistant bacteria among intensive care units patents at Ain Shams University Hospitals In Egypt- a retrospective study. J Egypt Public Health Assoc. 2021;96(1):7. doi: 10.1186/s42506-020-000658

11. Roy I, Jain A, Kumar M, Agarwal SK. Bacteriology of neonatal septicemia in a tertiary care hospital of Northern India. Indian J Med Microbiol. 2002;20(3):156159. doi: 10.1016/S0255-0857(21)03250-3

12. Meshram P, Gedam D, Ambhore N, Karayakarte R. Bloodstream Infections Prevalence in a tertiary care Institute, Central India. Indian J Microbiol Res. 2018;5(1):87-91. doi: 10.18231/2394-5478.2018.0018

13. Sharma M, Goel N, Chaudhary U, Aggarwai R, Arora DR. Bacteraemia in Children. Indian J Pediatr. 
2020;69(12):1029-1032. doi: 10.1007/BF02724380

14. Mehdinejah M, Khosravi AD, Morvaridi A. Study of Prevalence and antimicrobial susceptibility pattern of bacteria isolated from blood culture. J Bio Sci. 2009;9(3):249-253. doi: 10.3923/jbs.2009.249.253

15. Lee A, Mirrett S, Reller LB, Weinstein MP. Detection of blood stream infection in adults: How many blood culture are needed? J Clin Microbiol. 2007;45(11):35463548. doi: 10.1128/JCM.01555-07

16. Vasudeva N, Nirwan PS, Shrivastava P. Bloodstream Infections and antimicrobial sensitivity patterns in a tertiary care hospital of India. Ther Adv Infect Dis. 2016;3(5):119-127. doi: 10.1177/2049936116666983

17. Santella B, Folliero V, Pirofalo GM, et al. Sepsis - A Retrospective Cohort study of Bloodstream Infection. Antibiotics. 2020;9(12):851. doi: 10.3390/ antibiotics 9120851

18. Zenebe T, Kannan S, Yilma D, Beyene G. Invasive bacterial pathogens and their antibiotics susceptibility patterns in Jimma University Specialized Hospital, Jimma, Southwest Ethiopia. Ethiop J Health Sci. 2011;21(1):1-8. doi: 10.4314/ejhs.v21i1.69038

19. Al-Otaibi EF, Bukhari EE, Badr M, Alrabiaa AA. Prevalence and risk factors of Gram-negative bacilli causing bloodstream Infection in patients with malignancy. Saudi Med J. 2016;37(9):979-984. doi: 10.15537/smj.2016.9.14211

20. Karimzadeh I, Sadeghimanesh N, Mirzaee M, Saqheb MM. Evaluating the resistance pattern of Gram negative bacteria during three years at the nephrology ward of a referral hospital in Southwest of Iran. J Nephropathol. 2017;6(3):210-219. doi: 10.15171/ jnp.2017.35

21. Cole BK, Ilikji M, McCloskey CB, Charez-Bueno S.
Antibiotic resistance and molecular characterization of bacteraemia Escherichia coli isolated from new-borns in the United State. Plos ONE. 2019;14(7):e0219352. doi: 10.1371/journal.pone.0219352

22. Nagarjuna D, Mittal G, Dhanda SR, Gaind R, Yadar M. Alarming levels of antimicrobial resistance among sepsis patients admitted to ICU in a tertiary care hospital in India-a case control retrospective study. Antimicrob Resist Infect Control. 2018;7:150. doi: 10.1186/s13756-018-0444-8

23. Effah CY, Sun T, Liu S, Wu Y. Klebsiella pneumonia: an increasing threat to public health. Ann Clin Microbiol Antimicrob. 2020;19(1):1. doi: 10.1186/s12941-0190343-8

24. Clinical and Laboratory Standards Institute Performance Standards for Antimicrobial Susceptibility Testing Twenty - Fourth Informational Supplements. 2017; CLSI Documents M100-S27. Wayne, PA: Clinical and laboratory Standards Institute. www.clsi.org. Accessed $22 / 12 / 2020$

25. Singer M, Deutschman CS, Seymour CW, et al. The Third International Consensus Definitions for Sepsis and Septic Shock (Sepsis -3). JAMA. 2016;315(8):801810. doi: 10.1001/jama.2016.0287

26. Wisplinghoff $H$, Bischoff $T$, Tallent $S M$, Seifert $H$, Wenzel RP, Edmond MB. Nosocomial bloodstream infection in US hospitals. Analysis of 24,179 cases from a propective nationwide surveillance study. Clin infec Dis. 2014;39(3):309-317. doi: 10.1086/421946

27. Pyakurel $S$, Ansari M, Kattel $S$, et al. Prevalence of carbapenemase producing Klebsiella pneuminaie at a tertiary care hospital in Kathmandu, Nepel. Trop Med Health. 2021:49(1):78. doi: 10.1186/s41182-02100368-2 